\title{
The irp2 and fyuA genes in High Pathogenicity Islands are involved in the pathogenesis of infections caused by avian pathogenic Escherichia coli (APEC)
}

\author{
Jian Tu, Ting Xue, Kezong Qi, Ying Shao, Boyan Huang, \\ Xueyan Wang, Xiuhong Zhou
}

College of Animal Science and Technology, Anhui Agricultural University, Hefei 230036, PR China

\begin{abstract}
Avian pathogenic Escherichia coli (APEC) is a major bacterial infectious disease that may lead to local or systemic infections in chickens with clinical manifestations. The irp2-fyuA gene cluster has been confirmed to be the main genes involved in the synthesis of HPI. The objective of this study was to determine the influence of the irp 2 and fyu $A$ genes in the high pathogenicity island (HPI) of avian pathogenic Escherichia coli (APEC) on its pathogenicity by knocking out these genes. The $\triangle \mathrm{AE} 17$ (lacking irp2) and $\triangle \triangle \mathrm{AE} 17$ (lacking irp2 and fyuA) strains of APEC were constructed. The $\triangle \mathrm{AE} 17$ and $\triangle \Delta \mathrm{AE} 17$ strains showed significantly impaired capacity to adhere onto DF-1 cells. The $\mathrm{LD}_{50}$ results indicated that the virulence of the $\triangle \mathrm{AE} 17$ and $\triangle \triangle \mathrm{AE} 17$ strains was decreased in comparison with that of the AE17 strain. We concluded that the knock-out of the core HPI genes weakened APEC adhesion onto DF-1 cells, inhibited transcription of virulence genes, and reduced pathogenicity in chicks. The effects of genetic deletion of irp 2 and fyu $A$ on APEC were more severe than those produced by deletion of irp 2 only, indicating that irp 2 and fyu $A$ co-regulate APEC pathogenicity.
\end{abstract}

Key words: avian pathogenic Escherichia coli, irp2, fyuA, mutant strain, pathogenicity

\section{Introduction}

Avian pathogenic Escherichia coli (APEC) is a major bacterial infectious disease that may lead to local or systemic infections in birds such as chickens, ducks, and geese, with clinical manifestations including air sacculitis, perihepatitis, pericarditis, omphalitis, peritonitis, encephalitis, and salpingitis. APEC has seriously restricted the development of the poultry industry (Schouler et al. 2012). The pathogenicity of APEC is manifested as adhesion to host cells and enhanced transcription of virulence factors including adhesion, iron-binding proteins, antiserum factors, outer membrane proteins, and hemolysis (Han et al. 2013).

The Yersinia high pathogenicity island (HPI) was first identified by its association with pathogenic phenotypes in mice (Carniel et al. 1996). The HPI is a long chromosomal fragment containing several regulatory genes and the genes related to synthesis, 
uptake, and transport of siderophores such as yersiniabactin ( $\mathrm{Ybt}$ ), which is involved in the virulence and pathogenicity of Yersinia species (Rakin et al. 1999, Jacobi et al. 2001).

According to the gene sequence analysis, HPI could be divided into two evolutionary lines. The Yersinia pestis HPI and the Yersinia pseudotuberculosis HPI have a higher similarity in the nucleotide sequences, so the two bacterial HPI is an evolutionary system, referred to as YPs HPI. There are obvious differences in the HPI variable region sequences of Yersinia enterocolitica, so it is classified as another evolutionary system, referred to as Yen HPI. YPs HPI and Yen HPI are composed of two parts including the functional core area (about $30.5 \mathrm{~kb}$ ) and the variable region. The functional core area in HPI is highly conserved and up to $98-99 \%$ sequence homology. But the variable region in HPI is completely different between YPs HPI and Yen HPI (Carniel et al. 1996).

According to previous studies, HPI in Yersinia mediated iron uptake and regulation. At the same time, it is essential for the expression of mouse lethal protein. Although the gene names of the functional core area in YPs HPI or Yen HPI are different, the structure and location of these genes is similar. The gene location of the functional core area in YPs HPI is: 5'-asn-intB-irp9-irp8-irp7-irp6-ybtA-irp2-irp1-irp3-irp4-i rp5-fyua-3'. And the gene location of functional core area in Yen HPI is: 5-asn-int-ybtS-ybtX-ybtQybtP-ybtA-irp2-irp1-ybtU-ybtT-ybtE-psn-3'. In HPI, the irp2-irpl-irp3-irp4-irp5-fyua gene cluster has been confirmed to be the main genes involved in the synthesis of Ybt components, called the irp2-fyua gene cluster. At present, we have explored the function of irp1, irp2 and fyua. The irp1 and irp2 as the ferric uptake regulator gene, are expressed only in pathogenic Yersinia and closely related to the virulence. The two genes express the HMWP1(240KD) and HMWP2 (280KD) proteins, respectively. The HMWP1 protein is a multi enzyme complex and mediates the synthesis of no-ribosomal proteins or proteinoid. It is essential to the Ybt positive phenotype. The HMWP2 protein is mainly involved in siderophore and induces the expression of pesticin receptor and ironophore. The fyu $A$ gene encodes a $73.7 \mathrm{kD}$ iron-inhibition outer membrane protein which is the co-receptor between Bacterium bacteriocin and Bacillus yersini bacteriocin. The irp3 gene is $1098 \mathrm{bp}$ in size and contrary to the direction of transcription of irpl and irp2 genes. But the function of the gene is not clear. The irp 4 and irp5 genes are the same to direction of transcription of irpl and irp2 genes and may be related to Ybt synthesis. Studies have shown that the HPI is widely distributed in various intestinal pathogens. Clinical isolates of $93 \%$ of enteroaggregative E. coli, $27 \%$ of enteroinvasive E. coli, $5 \%$ of enteropathogenic E. coli, and $5 \%$ of enterotoxigenic E. coli carry the irp2-fyuA gene cluster of the HPI. The sequences of the irp2 and fyuA gene in $E$. coli are almost identical to those of Yersinia spp. (Schouler et al. 2005). The structural characteristics of the HPI in intestinal pathogens and Yersinia species are consistent and show high homology (Bach et al. 2000, Karch et al. 1999), indicating horizontal transmission of the HPI between species of bacteria. The irp 2 and $f y u A$ genes are the main structural genes in the irp2-fyu $A$ gene cluster, which is the core region of the HPI. The irp2 gene is the primary marker gene for HPI detection and an iron-regulating gene closely related to virulence (Carniel et al. 1996). The fy $A$ gene influences iron absorption and encodes an outer membrane receptor for Ybt, bacteriocin, and Pasteurella multocida toxin (Fetherston et al. 1994, Fetherston et al. 1995).

In this study, we used Red homologous recombination to knock out the irp 2 and $f y u A$ genes in HPI-positive APEC strains to construct the $\triangle \mathrm{AE} 17$ (lacking irp2) and $\Delta \Delta \mathrm{AE} 17$ (lacking irp2 and fyuA) strains to assess the influence of key HPI genes on the expression of APEC virulence genes, cell adhesion capacity, and pathogenicity in chicks. The study revealed that the $\Delta \mathrm{AE} 17$ and $\Delta \Delta \mathrm{AE} 17$ strains showed significantly impaired capacity to adhere onto DF-1 cells and transcript levels of luxs, pfs, tsh, iss, $о m p A$, and fim $C$ were significantly decreased compared to those of the AE17 strain. And the virulence of the $\triangle \mathrm{AE} 17$ and $\triangle \triangle \mathrm{AE} 17$ strains was decreased in comparison with that of the AE17 strain which was certified by chick $\mathrm{LD}_{50}$ results. This study laid the foundation for further investigation of the role of genes in the core region of the HPI in the pathogenesis of APEC.

\section{Materials and Methods}

\section{Bacterial strains and culture conditions}

AE17 was isolated from ducks in An'hui Province, China in 2008. AE17 was identified as E. coli stains by biochemical identification and 16S rDNA analysis and then identified as the serotype $\mathrm{O} 2$ strain of $E$. coli by serological identification. AE17 was grown on liquid LB medium or on $1.2 \%$ solid LB medium at $37^{\circ} \mathrm{C}$. Ampicillin (100 mg/ml) and chloramphenicol (34 $\mathrm{mg} / \mathrm{ml}$ ) were added to the medium for the antibiotics group. 
Table 1. Primers used in the gene deletion.

\begin{tabular}{|c|c|c|}
\hline Primer & Primer sequence & Size (bp) \\
\hline irp2-UF & GACTCCCACTGCTGTTGC & 760 \\
\hline irp2-UR & GTCAAGCAACC_CGGAATTCCAGCGAATCCTGAGATGGT & \\
\hline irp2-DF & 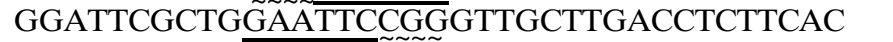 & 1114 \\
\hline irp2-DR & ATAGCCGACCTTTCTGTT & \\
\hline pkD3- EcoRI- F & CCCGGAATTCTGTAGGCTGGAGCTGCTTCGA & 1013 \\
\hline pkD3- EcoRI- R & 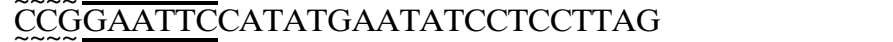 & \\
\hline irp2-inF & 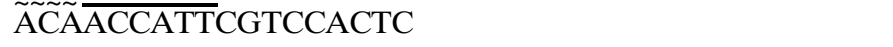 & \\
\hline irp2-inR & GTTATCCAGACACAGCCA & \\
\hline irp2-outF & TTTAAACGCGGGATGTA & \\
\hline irp2-outR & GAAGGGTGGGTGAAGAG & \\
\hline fyuAUF & TCAGCCAACAACGTCTCG & 551 \\
\hline fyuA-UR & CCCTGGTCGCC_GCGTCGACCGCAGTTTCACATTCCCTAT & \\
\hline fyuA-DF & 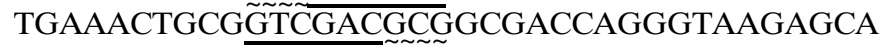 & 651 \\
\hline fyuA-DR & GGTTATCGACATAGACGGAAAT & \\
\hline pkD3- SalI - F & CGCGTCGACTGTAGGCTGGAGCTGCTTCGA & 1013 \\
\hline pkD3- SalI - R & 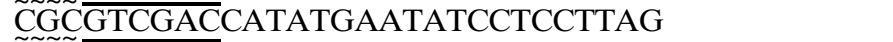 & \\
\hline fyuA-inF & $\tilde{\text { GCC }} \tilde{\tilde{C} A T G T G G G A T G G A A T G}$ & \\
\hline fyuA-inR & TGCCAGGTCAGGTCACTGTATG & \\
\hline fyuA -outF & AATGCCCAGACTTCACAGC & \\
\hline fyuA -outR & CGACGGTCGAACAGGTTA & \\
\hline
\end{tabular}

\section{Construction and identification of the irp2 mutant strain $\triangle \mathrm{AE} 17$}

Red homologous recombination was used to construct the $\triangle \mathrm{AE} 17$ strain lacking irp2. The homology arms upstream and downstream of the irp2 gene were amplified and performed to amplify the irp2-Up-Down fragment with irp2-Up and irp2-Down as templates. The irp2-Up-Down fragment was cloned to the zero background vector pZero-back (Tiangen, China) to form recombinant plasmid pZero-Up-Down. The fragment was amplified from the pKD3 plasmid (Yale University Preservation Center, New Haven, CT, USA) flanked by EcoRI restriction enzyme digestion sites. The recovered product and the plasmid were simultaneously digested by EcoRI restriction enzyme (Promega, Madison, WI, USA) and connected to form pZero-Up-cat-Down. Taking pZero-Up-cat-Down as a template, the irp2-UF and irp2-DR primers were used to amplify the large fragment irp2-Up-cat-Down for Red homologous recombination. The purified product was added to competent cells of strain AE17 containing the pKD46 plasmid (Yale University Preservation Center, New Haven, CT, USA). Electroporation was performed using a Bio-Rad Gene Pulser Xcell Electroporation System $(200 \Omega, 25 \mu \mathrm{F}, 2.5 \mathrm{kV})$ at $37^{\circ} \mathrm{C}$ overnight (Bio-Rad Laboratories, Hercules, CA, USA). Then the cells were inoculated in $100 \mathrm{ml} \mathrm{LB}$ medium (Sangon Biotech, China) containing 100 $\mathrm{mg} / \mathrm{ml}$ ampicillin at a rate of 1:100. After electroporation and culture, the cells were spread on a chloramphenicol-coated plate. Two pairs of primers
irp2-inF/irp2-inR and irp2-outF/irp2-outR were used to identify chloramphenicol-resistant cells to detect whether the irp2 gene of AE17 was knocked out.

The plasmid (Yale University Preservation Center, New Haven, CT, USA) was electroporated into the chloramphenicol-resistant recombinant cells. The LB plate showing ampicillin/chloramphenicol double resistance was used to culture the recombinants at $28^{\circ} \mathrm{C}$. The positive transformants were picked, inoculated in antibiotic-free LB medium, and cultured at $42^{\circ} \mathrm{C}$ overnight. FLP recombinase expression was induced thermally. By recombination, the sequence between the FRT sites was removed, preserving only one FRT site. PCR using the irp2-outF/irp2-outR primers was carried out for verification.

\section{Construction and identification of the irp2/fyuA mutant strain $\triangle \triangle \mathrm{AE} 17$}

The $\triangle \Delta \mathrm{AE} 17$ strain lacking $f y u A$ and irp 2 was created from the $\triangle \mathrm{AE} 17$ strain by the same method used for knocking out the irp2 gene. The homology arms upstream and downstream (fyuA-Up, 551 bp; fyu $A$-Down, $651 \mathrm{bp)}$ of the fyu $A$ gene were amplified with the fyuA-UF/fyuA-UR and fyuA-DF/fyuA-DR primer pairs, respectively. Overlap PCR was performed to amplify the fyuA-Up-Down fragment with the fy $u A$-UF and fy $u A$-DR primers. The PCR product was cloned into the zero background vector pZero-back (Tiangen, China) to form the pZero-Up-Down plasmid. The chloramphenicol-resistant fragment was amplified from the pKD3 plasmid 
Table 2. Primers used in the Real time PCR.

\begin{tabular}{|c|c|c|c|}
\hline Gene & Primer & Primer sequence ( $5^{\prime}$ to $\left.3^{\prime}\right)$ & Size (bp) \\
\hline $\begin{array}{l}\text { dnaE } \\
\text { dnaE }\end{array}$ & $\begin{array}{l}\text { dnaE-F } \\
\text { dnaE-R }\end{array}$ & $\begin{array}{l}\text { GATTGAGCGTTATGTCGGAGGC } \\
\text { GCCCCGCAGCCGTGAT }\end{array}$ & 80 \\
\hline $\begin{array}{l}\text { luxs } \\
\text { luxs }\end{array}$ & $\begin{array}{l}\text { luxs-F } \\
\text { luxs-R }\end{array}$ & $\begin{array}{l}\text { ACGCCATTACCGTTAAGATG } \\
\text { AGTGATGCCAGAAAGAGGGA }\end{array}$ & 81 \\
\hline $\begin{array}{l}\text { pfs } \\
\text { pfs }\end{array}$ & $\begin{array}{l}\text { pfs-F } \\
\text { pfs-R }\end{array}$ & $\begin{array}{l}\text { CGGCAACAGCCAGGAACTCA } \\
\text { GCGAAAATCCGCCACAACTT }\end{array}$ & 169 \\
\hline $\begin{array}{l}\text { tsh } \\
\text { tsh }\end{array}$ & $\begin{array}{l}\text { tsh-F } \\
\text { tsh-R }\end{array}$ & $\begin{array}{l}\text { GCACGAACTGGGAAGTATGGA } \\
\text { GGCATAGAAACCACCACCCC }\end{array}$ & 118 \\
\hline $\begin{array}{l}\text { ibeA } \\
\text { ibeA }\end{array}$ & $\begin{array}{l}\text { ibeA-F } \\
\text { ibeA-R }\end{array}$ & $\begin{array}{l}\text { TTGTTTTGGCGGAATGATG } \\
\text { CATTGATTTTGCCGTTTCTTCT }\end{array}$ & 118 \\
\hline $\begin{array}{l}\text { stx2f } \\
\text { stx2f }\end{array}$ & $\begin{array}{l}\text { stx2f-F } \\
\text { stx2f-R }\end{array}$ & $\begin{array}{l}\text { GTGTAAAACTACGCCATCCG } \\
\text { AAGCCCAGAACCAGACTCCC }\end{array}$ & 186 \\
\hline $\begin{array}{l}\text { iss } \\
\text { iss }\end{array}$ & $\begin{array}{l}\text { iss-F } \\
\text { iss- } \mathrm{R}\end{array}$ & $\begin{array}{l}\text { CCGACAGCAGTAACACCAAAGG } \\
\text { TTCTGCACCGCCAACAAATT }\end{array}$ & 105 \\
\hline $\begin{array}{l}\text { ompa } \\
\text { ompa }\end{array}$ & $\begin{array}{l}\text { ompa-F } \\
\text { ompa-R }\end{array}$ & $\begin{array}{l}\text { TCCAGAGCAGCCTGACCTTC } \\
\text { GCTGAGCCTGGGTGTTTCCT }\end{array}$ & 152 \\
\hline $\begin{array}{l}\text { vat } \\
\text { vat }\end{array}$ & $\begin{array}{l}\text { vat-F } \\
\text { vat-R }\end{array}$ & $\begin{array}{l}\text { TAACGGTATCCGACTTCTGC } \\
\text { ATGGGGTGGTGGTTTCTATG }\end{array}$ & 171 \\
\hline $\begin{array}{l}\text { fimc } \\
\text { fimc }\end{array}$ & $\begin{array}{l}\text { fimc-F } \\
\text { fimc-R }\end{array}$ & $\begin{array}{l}\text { GCCGATGGTGTAAAGGATGG } \\
\text { АACTTTCCCGATCCTGTGGC }\end{array}$ & 127 \\
\hline
\end{tabular}

using the primers pkD3-SalI-F/pkD3-SalI-R flanked by SalI restriction enzyme digestion sites. The recovered product and the recombinant plasmid pZeroUp-Down were simultaneously digested by the SalI enzyme (Promega, Madison, WI, USA). The digested chloramphenicol-resistant fragment was connected with pZero-Up-Down to form pZero-Up-cat-Down. Using pZero-Up-cat-Down as a template, the primers $f y u A$-UF and fyuA-DR were used to amplify the fragment fyuA-Up-cat-Down (2215 bp) for Red homologous recombination. The sequences of the primers are shown in Table 1.

The constructed fragment fyu $A$-Up-cat-Down was electroporated into $\triangle \mathrm{AE} 17$ cells containing pKD46. Two pairs of primers $(f y u A$-inF/fy $u A$-inR and $f y u A$-outF/fyu $A$-outR) were used for the identification of chloramphenicol-resistant recombinants.

\section{Assay for bacterial adherence}

The AE17, $\triangle \mathrm{AE} 17$, and $\triangle \Delta \mathrm{AE} 17$ strains were cultured in liquid $\mathrm{LB}$ medium at $37^{\circ} \mathrm{C}$ until reaching the logarithmic phase. The bacterial solution was centrifuged at $6000 \times g$ for $5 \mathrm{~min}$ at $4^{\circ} \mathrm{C}$. The cells obtained were rinsed 3 times with sterile PBS, antibiotic-free DMEM was added (Thermo Scientific, Pittsburgh, PA, USA), and they were shaken well. DF-1 cells (DF-1 is an immortalized cell line of chicken embryo fibroblasts) were cultured in DMEM nutrient solution containing $10 \%$ fetal calf serum and antibiotics. The cells were added to a 24-well plate and cultured at $37^{\circ} \mathrm{C}$ in an incubator with $5 \% \mathrm{CO} 2$ and saturated humidity. When the well was overgrown with cells, the medium was removed and the cells were rinsed 3 times with sterile PBS. Each of the experimental groups with three replicates.

After rinsing, the $\mathrm{AE} 17, \triangle \mathrm{AE} 17$, and $\triangle \triangle \mathrm{AE} 17$ strains (multiplicity of infection $(\mathrm{MOI})=200$ ) were added to the DF-1 cells. The control group of DF-1 cells was exposed to an equal volume of antibiotic-free DMEM. Each group was assessed in 3 replicates wells. Low-velocity centrifugation was performed to bind the bacteria to the cells, and the cells were cultured in an incubator at $37^{\circ} \mathrm{C}$ for $1.5 \mathrm{hr}$. The cells were rinsed 5 times with sterile PBS and $200 \mu \mathrm{L} 0.1 \%$ pancreatin was added to each well to disrupt the cells at room temperature for $10 \mathrm{~min}$. The plate count method was used to count the number of bacteria in the cytosol. The colonies were counted after the cells were cultured on $\mathrm{LB}$ plates at $37^{\circ} \mathrm{C}$ overnight.

\section{RNA isolation, reverse transcription and quantitative real-time PCR}

The virulence genes luxs, $p f s, t s h$, ibe A, stx $2 f$, iss, ompa, vat, and fimc of avian pathogenic $E$. coli were selected for quantitative real-time PCR (qPCR). The housekeeping gene $d n a E$ was used to normalize the 
amount of RNA across the samples and to assess the reverse transcription efficiency of cDNA(Table 2). Total RNA of AE17, $\triangle \mathrm{AE} 17$, and $\triangle \triangle \mathrm{AE} 17$ stains was extracted from the 3 groups of bacteria separately. The RNA concentration and purity were detected by a Nucleic Acid Protein Detector (ZHD-3, Nanjing Chishun Science \& Technology Co., Ltd., Nanjing, China). The DNA-freeTM kit (Ambion ${ }^{\circledR}$, Life Technologies, Carlsbad, CA, USA) was used to digest the DNA in the RNA solution. The RNA was uniformly mixed with $0.1 \mathrm{~V}$ DNase I buffer and $1 \mu \mathrm{L}$ of DNase I, reacted in a $37^{\circ} \mathrm{C}$ water bath for $30 \mathrm{~min}$, and 0.1 $\mathrm{V}$ DNase inactivation reagent was added. The solution was centrifuged at $1000 \times g$ for $2 \mathrm{~min}$, and the supernatant containing the purified RNA was collected. Next, $1 \mu \mathrm{l}$ of random primerwas added to $2 \mu \mathrm{g}$ RNA. The RNA was degenerated and exposed to an ice bath for $1 \mathrm{~min}$, after which $1 \mu \mathrm{l}$ of M-MLV, $5 \mu \mathrm{l}$ of 5x M-MLV reaction buffer, $4 \mu \mathrm{l}$ dNTP mixture, and $1 \mu \mathrm{l}$ of RNase inhibitor were added. RNase-free water was added. The mixture was reacted at $42^{\circ} \mathrm{C}$ for $1 \mathrm{~h}$ and stored at $-40^{\circ} \mathrm{C}$. The cDNA was diluted stepwise and used to plot a 5-point relative standard curve. Reaction system $(20 \mu \mathrm{l}): 10 \mu \mathrm{l}$ SYBR Premix Ex Taq (Promega, Madison, WI, USA), $0.5 \mu \mathrm{l}$ Primer $1\left(10 \mathrm{pmol} \cdot \mathrm{L}^{-1}\right), 0.5 \mu \mathrm{l}$ Primer $2\left(10 \mathrm{pmol} \cdot \mathrm{L}^{-1}\right), 1 \mu \mathrm{l}$ template, $8 \mu l$ ddH2O. Each sample had 3 replicates. A melting curve was obtained to analyze the specificity of the primers. Virulence analysis was performed with the $2-\Delta \Delta C T$ method, and the transcript levels of virulence genes were calculated.

\section{Animals}

One-day-old Roman chicks, purchased from Changfeng County, Hefei City, An'hui Province, were reared at $28-30^{\circ} \mathrm{C}$ with free access to food and water (complete diet without antibiotics) and 12-hr illumination every day. Healthy 7-day-old chicks were selected for the experiment.

\section{Determination of the lethal dose of the bacteria $\left(\mathbf{L D}_{50}\right)$}

The AE17, $\triangle \mathrm{AE} 17$, and $\triangle \Delta \mathrm{AE} 17$ strains were separately added to liquid LB medium and cultured at $37^{\circ} \mathrm{C}$ until reaching the logarithmic phase. The cells were collected, rinsed 3 times with sterile PBS, and resuspended. The number of bacteria was regulated and multiple proportion dilution was performed. Each strain was inoculated into 5 groups $(\mathrm{n}=8$ each) of 7-day-old Roman chicks. The dosages of the AE17 and $\triangle \mathrm{AE} 17$ strains in the 5 groups were $2 \times 10^{8}$
$\mathrm{CFU} /$ chick, $2 \times 10^{7} \mathrm{CFU} /$ chick, $2 \times 10^{6} \mathrm{CFU} /$ chick, $2 \times 10^{5} \mathrm{CFU} /$ chick, and $2 \times 10^{4} \mathrm{CFU} /$ chick in the 5 groups. The dosages of the $\triangle \triangle \mathrm{AE} 17$ strain in the 5 groups were $2 \times 10^{10} \mathrm{CFU} /$ chick, $2 \times 10^{2} \mathrm{CFU} /$ chick, $2 \times 10^{8} \mathrm{CFU} /$ chick, $2 \times 10^{7} \mathrm{CFU} /$ chick, and $2 \times 10^{6}$ CFU/chick. The control group was treated with sterile PBS. The chicks were infected by intramuscular injection. After injection, the chicks were observed for 7 days and the survival status was recorded. The survival rate was used to calculate the $50 \%$ lethal dose $\left(\mathrm{LD}_{50}\right)$ of the 3 strains based on the Reed-Muench method.

\section{Results}

\section{Identification of the mutant strain $\triangle \mathrm{AE} 17$ and $\triangle \triangle \mathrm{AE} 17$}

PCR confirmed that the irp2 knockout strain $\triangle$ AE17 was constructed successfully (Fig. 1). The pKD46 plasmid and the target fragment fyu $A$-up-cat-down were successively electroporated into the $\triangle \mathrm{AE} 17$ strain. PCR was performed to verify the construction of the irp2/fyuA knockout strain $\triangle \triangle \mathrm{AE} 17$ (Fig. 2).

\section{Comparison of AE17, $\triangle \mathrm{AE} 17$, and $\triangle \triangle \mathrm{AE} 17$ adhesion to DF-1 cells}

The adhesion capacity of the AE17, $\triangle \mathrm{AE} 17$ and $\triangle \triangle \mathrm{AE} 17$ strains was $9.04 \times 10^{4}, 5.97 \times 10^{4}$, and 4.84 $\times 10^{4} \mathrm{CFU} /$ well, respectively. As shown in Figure 3, in comparison with the AE17 strain, the adhesion capacity of the $\triangle \mathrm{AE} 17$ and $\triangle \Delta \mathrm{AE} 17$ strains was significantly decreased $(\mathrm{P}<0.01)$. The adhesion capacity of $\triangle \triangle \mathrm{AE} 17$ strain was significantly lower than that of the $\triangle \mathrm{AE} 17$ strain $(\mathrm{P}<0.05)$.

\section{Transcriptional analysis of virulence genes in the AE17, $\triangle \mathrm{AE} 17$, and $\triangle \triangle \mathrm{AE} 17$ strains}

The melting curve analysis showed that the primers for the reference gene $d n a E$ and the virulence genes luxs, pfs, tsh, ibe A, stx2f, iss, ompA, vat, and fim $C$ had high specificity. RT real-time PCR results indicated that the $\triangle \mathrm{AE} 17$ strain had significantly decreased levels of the luxs, iss, ompA, and fimC transcripts $(\mathrm{P}<0.01)$, which were $0.32,0.27,0.33$, and 0.25 times as abundant as those of the AE17 strain, respectively. In the $\triangle \mathrm{AE} 17$ strain, $p f s$ mRNA abundance was significantly decreased to 0.79 times than that of the AE17 strain. However, the abundance of 


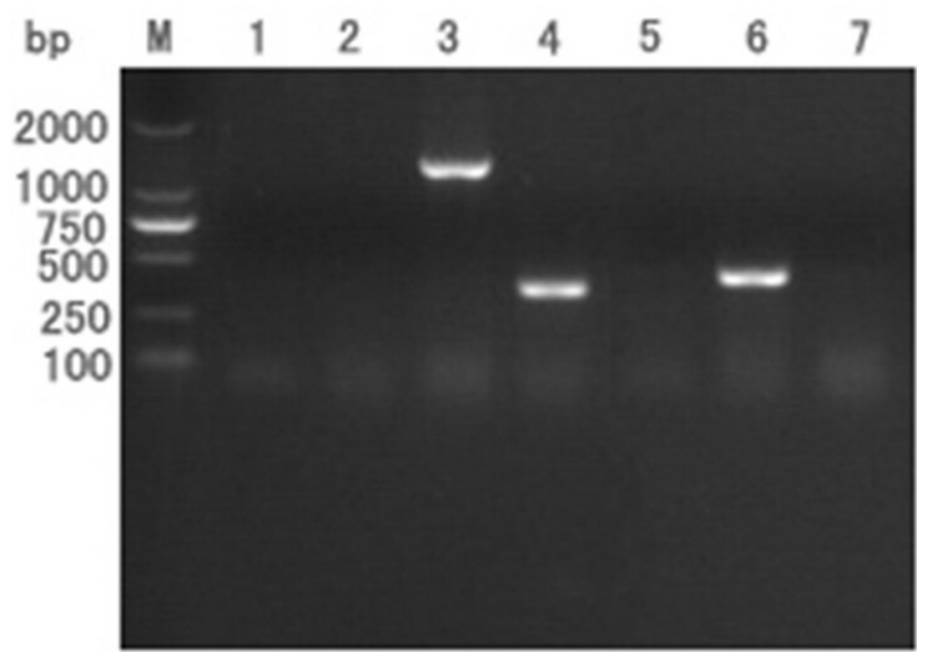

Fig. 1. PCR analysis of the mutant strain $\triangle \mathrm{AE} 17$. Lane 1: Negative control. Lane 2: The mutant strain $\triangle \mathrm{AE} 17$, mutant strain showed no PCR product using primers irp2-outF/irp2-outR. Lane 3: The $\Delta$ AE17, mutant strain $\triangle \mathrm{AE} 17$ showed a 1282 bp PCR product (Containing the chloramphenicol resistance gene) using primers irp2-outF/irp2-outR. Lane 4:The $\Delta \mathrm{AE} 17$ mutant strain, $\triangle \mathrm{AE} 17$ showed a $281 \mathrm{bp}$ PCR product (Does not containing the chloramphenicol resistance gene) using primers irp2-outF/irp2-outR. Lane 5: Negative control. Lane 6: The mutant strain $\triangle \mathrm{AE} 17$, mutant strain showed a 418 bp PCR product using primers irp2-inF/irp2-inR. Lane 7: The mutant strain $\triangle \mathrm{AE} 17$, mutant strain showed no PCR product using primers irp2-inF/irp2-inR.

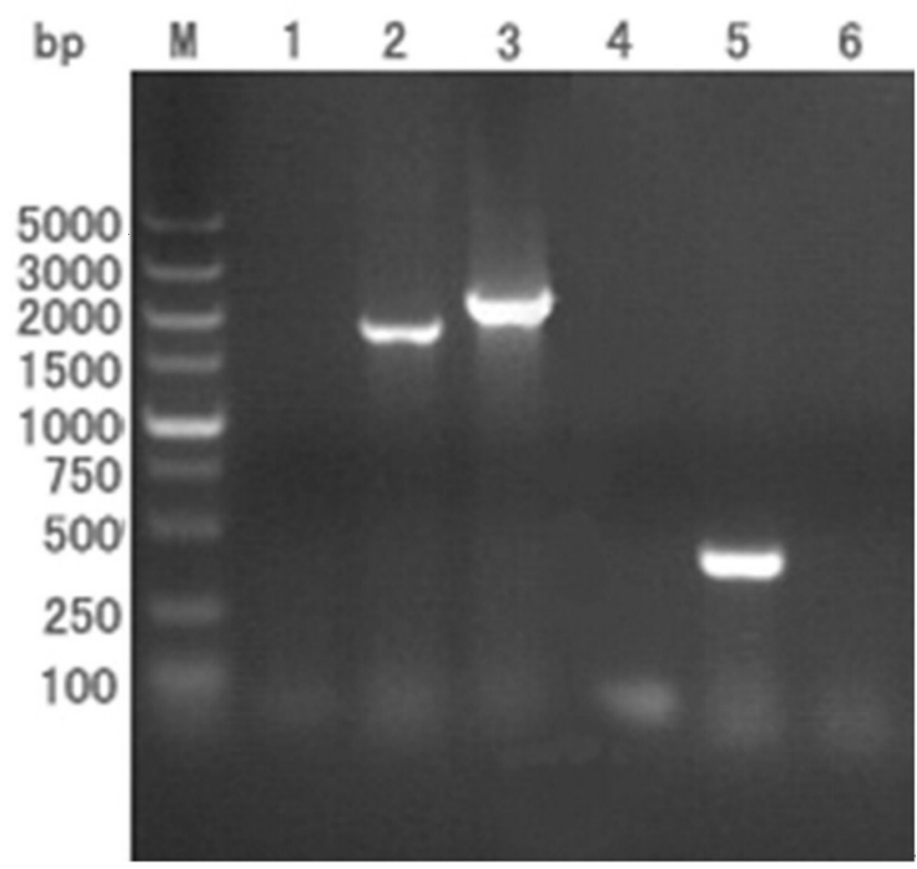

Fig. 2. PCR analysis of the mutant strain $\Delta \Delta \mathrm{AE} 17$. Lane 1: Negative control. Lane 2: The mutant strain $\Delta \Delta \mathrm{AE} 17$ mutant strain showed a $1865 \mathrm{bp}$ PCR product using primers fyu $A$-outF/ fyu $A$--outR. Lane 3: The mutant strain $\triangle \Delta \mathrm{AE} 17$ mutant strain showed a 2316 bp PCR product (Containing the chloramphenicol resistance gene) using primers fyuA -outF/ fyu $A$-outR. Lane 4: Negative control. Lane 5: The mutant strain $\triangle \triangle \mathrm{AE} 17$ mutant strain showed a $410 \mathrm{bp}$ PCR product using primers fyu $A$-inF/fyu $A$-inR. Lane 6: The mutant strain $\triangle \Delta \mathrm{AE} 17$ mutant strain showed no PCR product using primers fyuA-inF/fyuA-inR. 


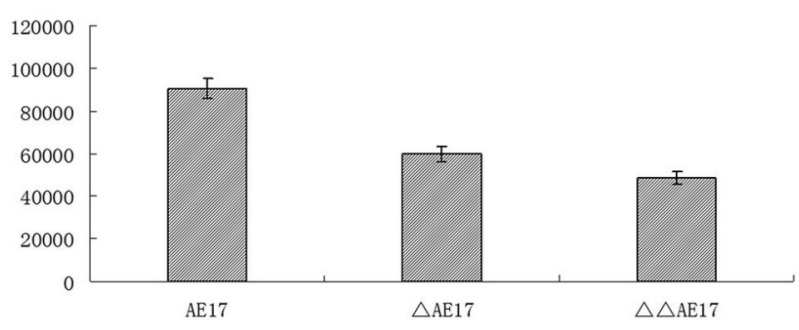

Fig. 3. Adherence assay. Adherence to DF-1 cells by the $\triangle \mathrm{AE} 17$ and $\triangle \triangle \mathrm{AE} 17$ mutant strains were significantly reduced compared with AE17 strain. The ability of $\triangle \mathrm{AE} 17$ adhesion of the DF-1 cells was significantly reduced decreased, in comparison with that of the $\triangle \triangle \mathrm{AE} 17$ strain. The columns represent the means and standard deviations.

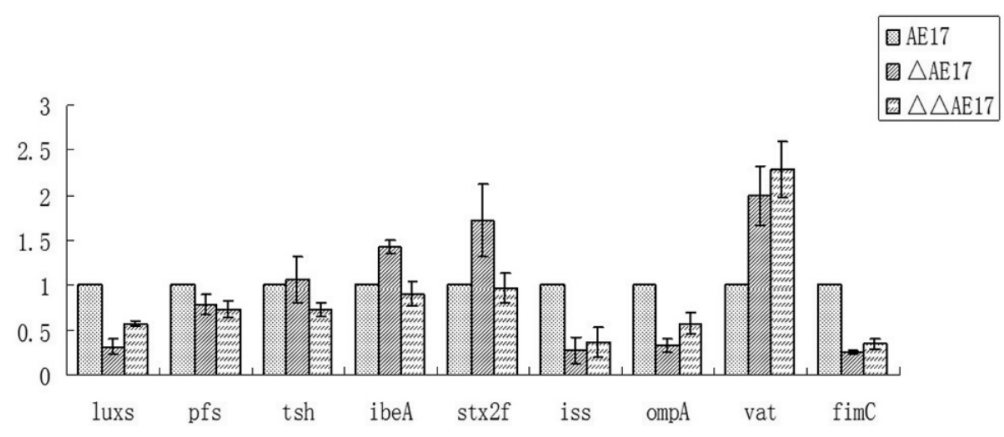

Fig. 4. Differences were found in virulence gene expression in the $\triangle \mathrm{AE} 17$ and $\triangle \triangle \mathrm{AE} 17$ strains. The three columns show the virulence gene mRNA transcript levels of the AE17, $\triangle \mathrm{AE} 17$, and $\triangle \triangle \mathrm{AE} 17$ strains. The relative expression level of each gene was normalized to that of $d n a E$. Results are shown as relative expression ratios compared to the expression of each transcript in the wild-type strain AE17. Each value represents the mean and standard deviation.

stx2f mRNA in the $\triangle \mathrm{AE} 17$ strain was significantly increased to 1.72 times than that of the AE17 strain. In the $\triangle \mathrm{AE} 17$ strain, abundance of $i b e A$ and $v a t$ mRNA was increased significantly to 1.43 and 2.00 times than that of the AE17 strain, respectively. There was no significant change in the transcript levels of $t s h$. The $\triangle \triangle \mathrm{AE} 17$ strain showed significant decreases in the levels of luxs, pfs, tsh, iss, ompA, and fimC transcripts, which were $0.57,0.73,0.73,0.37,0.57$, and 0.35 times as abundant as those of the AE17 strain, respectively $(\mathrm{P}<0.01)$. As shown in Figure 4, there were no significant differences in the levels of $i b e A$ and stx $2 f$ mRNA transcripts in the $\triangle \triangle \mathrm{AE} 17$ and AE17 strains. The results shown that the irp2 and fyuA genes in high pathogenicity island may induce transcript levels of luxs, $p f s$, tsh, iss, ompA, and fimC significantly decreased in avian Escherichia coli.

\section{AE17, $\triangle \mathrm{AE} 17$, and $\triangle \triangle \mathrm{AE} 17$ median lethal dose $\left(\mathbf{L D}_{\mathbf{5 0}}\right)$ determination}

After the Roman chicks were injected with the bacteria for $24 \mathrm{hr}$, they began to show lassitude, inappetence, and hypothermia. After $36 \mathrm{hr}$, there was some mortality in the APEC-infected groups, but not in the control group. Karber's method was modified to determine the $\mathrm{LD}_{50}$ values for the APEC strains. The $\mathrm{LD}_{50}$ values of the AE17, $\triangle \mathrm{AE} 17$, and $\triangle \Delta \mathrm{AE} 17$ strains were $1.5 \times 10^{6}, 5.1 \times 10^{7}$, and $7.5 \times 10^{8}$ CFU/chick, respectively. In comparison with the AE17 strain, the virulence of the $\triangle \mathrm{AE} 17$ and $\triangle \triangle \mathrm{AE} 17$ strains reduced by $34-$ and $500-$ fold. The $\triangle \triangle \mathrm{AE} 17$ strain was 14.7-fold less virulent than the $\triangle \mathrm{AE} 17$ strain (Tables 3 and 4). The results shown that the irp 2 and fyu $A$ genes in high pathogenicity island may induce the virulence of avian Escherichia coli decreased which was certified by chick $\mathrm{LD}_{50}$ results.

\section{Discussion}

By using Red homologous recombination, the irp2 mutant and the irp2/fyuA mutant strains were constructed successfully in this study. These mutants served as the foundation for further study of the relationship between the Yersinia HPI and APEC pathogenicity. Red recombination technology is a genetic engineering technology based on the Red recombinase of the bacteriophage $\lambda$ and in vivo homologous recombination. Using 3' recombinant bacteriophage $\lambda$ proteases, this technology realizes homologous recombination between DNA fragments and the chromosome in vitro (Muyrers et al. 2001, Poteete et al. 2001). 
Table 3. Mortality of E. coli-infected chicks.

\begin{tabular}{cccc}
\hline Group & Number of experimental animals & Number of dead animals & Mortality rate $(\%)$ \\
\hline Positive & 20 & 14 & 70 \\
AE-a1 & 20 & 8 & 40 \\
AE-t & 20 & 0 & 0 \\
Control & 20 & 0 & 0 \\
\hline
\end{tabular}

Table 4. The comparison of virulence of AE17 and $\Delta \Delta \mathrm{AE} 17$.

\begin{tabular}{|c|c|c|c|c|c|c|c|}
\hline Group & & & & & & & $\begin{array}{c}\mathrm{LD}_{50} \\
(\mathrm{CFU})\end{array}$ \\
\hline AE17 & $\begin{array}{c}2 \times 10^{8} \\
8 / 8\end{array}$ & $\begin{array}{c}2 \times 10^{7} \\
7 / 8\end{array}$ & $\begin{array}{c}2 \times 10^{6} \\
5 / 8\end{array}$ & $\begin{array}{c}2 \times 10^{5} \\
1 / 8\end{array}$ & $\begin{array}{c}2 \times 10^{4} \\
0 / 8\end{array}$ & $\begin{array}{c}\text { Saline } \\
0 / 8\end{array}$ & $1.5 \times 10^{6}$ \\
\hline$\Delta \Delta \mathrm{AE} 17$ & $\begin{array}{l}10^{10} \\
8 / 8\end{array}$ & $\begin{array}{l}10^{9} \\
5 / 8\end{array}$ & $\begin{array}{l}10^{8} \\
0 / 8\end{array}$ & $\begin{array}{l}10^{7} \\
0 / 8\end{array}$ & $\begin{array}{l}10^{6} \\
0 / 8\end{array}$ & $\begin{array}{c}\text { Saline } \\
0 / 8\end{array}$ & $7.5 \times 10^{8}$ \\
\hline
\end{tabular}

As an essential nutrient for the microbial growth, iron is an indispensable bacterial growth factor and a regulator of bacterial virulence factor expression. Many bacteria have the ability to acquire iron from host species, and this ability is a major influence factor on their pathogenesis. The HPI encodes genes that uptake iron from the host through Ybt and then affects virulence of APEC. As important structural genes in the core region of the HPI, irp 2 and fyu $A$ play key roles in the iron uptake system encoded by the HPI of E. coli (Kurmanova et al. 2007). Therefore, it was speculated that deletion of irp 2 and $f y u A$ may influence the iron uptake capacity of APEC strains and the expressions of some virulence factors. This study showed that, in the $\triangle \mathrm{AE} 17$ strain, the transcription of luxs, iss, ompA, fimC, and $p f s$ was down-regulated, and the transcription of stx $2 f$ and $i b e A$ genes was up-regulated. In the $\triangle \triangle \mathrm{AE} 17$ strain, the transcription of luxs, pfs, tsh, iss, ompA, and fimC was down-regulated. Notably, compared with the single knockout $\triangle \mathrm{AE} 17$ strain, the double knockout $\triangle \triangle \mathrm{AE} 17$ strain showed more down-regulated virulence genes. Although transcription of stx $2 f$ and $i b e A$ was up-regulated in the $\triangle \mathrm{AE} 17$ strain, there was no significant difference in the expression of these genes between the $\triangle \mathrm{AE} 17$ and AE17 strains. The decreased transcription of virulence gene indicated weakened AE17 pathogenicity, and thus the double HPI gene knockout impaired pathogenicity more strongly than the single HPI gene knockout.

The capacity of bacteria to adhere to host cells or the surfaces of other organs is vital for their colonization and survival (Schouler et al. 2005). The analysis of adhesion capacity indicated that, compared with the AE17 strain, the capacity to adhere to the cells of the $\triangle \mathrm{AE} 17$ and the $\triangle \triangle \mathrm{AE} 17$ was reduced. Adhesion, an important virulence factor of pathogens, is localiz- ed on the bacterial surface. Adhesin can bind to the surface receptors of the host cell to escape scavenging, and it is thus very important in the pathogenic process (Antao et al. 2009). The fimC and ompA genes encode adherence factors that influence $E$. coli adherence to host cells (Jones et al. 1993). These results confirmed that decreased fimC and ompA transcription may reduce the adhesion capacity of APEC.

$\mathrm{LD}_{50}$ values intuitively and accurately reflect bacterial virulence. $\mathrm{LD}_{50}$ is inversely proportional to the bacterial virulence; larger $\mathrm{LD}_{50}$ values indicate less virulence. Our $\mathrm{LD}_{50}$ results showed that the AE17 strain showed the strongest virulent in the tested strains. Gene deletion significantly decreased virulence, and double gene-deleted strain showed the weakest virulent. After the determination of $\mathrm{LD}_{50}$ values of the $\mathrm{AE} 17, \triangle \mathrm{AE} 17$, and $\triangle \Delta \mathrm{AE} 17$ strains, the difference in the pathogenicity of these strains in chicks was studied.

In this work, we report the successful construction of the irp2 knockout APEC strain and the irp2/fyuA knockout APEC strain. Deletion of the HPI core gene irp2 reduced transcription of virulence genes, capacity to adhere to DF-1 cells, and pathogenicity in chicks, and the deletion of irp 2 and fyu $A$ resulted magnified these effects. This study might lay the foundation for further investigation of the genes in the core region of the HPI in the pathogenesis of APEC

\section{References}

Antao EM, Wieler LH, Ewers C. (2009) Adhesive threads of extraintestinal pathogenic Escherichia coli. Gut Pathog 1: 22.

Bach S, Almeida A, Carniel E. (2000) The Yersinia high-pathogenicity island is present in different members of the family Enterobacteriaceae. FEMS Microbiol Lett 183: 289-294. 
Carniel E, Guilvout I, Prentice M. (1996) Characterization of a large chromosomal :high pathogenicity island; in biotype 1B Yersinia enterocolitica. J Bacteriol 178: 6743-6751.

Fetherston JD, Lillard JJW, Perry RD. (1995) Analysis of the pesticin receptor from Yersinia pestis, role in iron-deficient growth and possible regulation by its siderophore. Bacteriol. 177: 1824-1833.

Fetherston JD, Perry RD. (1994) The pigmentation locus of Yersinia pestis is flanked by an insertion sequence and includes the structural genes for pesticin sensitivity and HMWP2. Mol Microbiol 13: 697-708.

Han XG, Bai H, Lei L, et al. (2013) The luxS gene functions in the pathogenesis of avian pathogenic Escherichia coli. Microbial Pathog 6: 21-27.

Jacobi CA, Gregor S, Rakin A, Heesemann J. (2001) Expression Analysis of the Yersiniabactin Receptor Gene fyuA and the Heme Receptor hemR of Yersinia enterocolitica In Vitro and In Vivo Using the Reporter Genes for Green Fluorescent Protein and Luciferase. Infect Immun 69: 7772.

Jones CH, Pinkner JS, Nicholes AV, et al. (1993) Fim C is a periplasmic PapD-like chaperone that directs assembly of type 1 pili in bacteria. Proc Natl Acad Sci USA 90: 8397-8401.

Karch H, Schubert S, ZhangD. et al. (1999) A genomic island, termed high-pathogenicity island, is present in cer- tain non-O157 Shiga toxin-producing Escherichia coli clonal lineages. Infect Immun 67: 5994-6001.

Kurmanova A, Llorente A, Polesskaya A, et al. (2007) Structural requirements for furin-induced cleavage and aetivation of Shiga toxin. Biochem Biophys Res Commun 357: 144-149.

Muyrers JP, Zhang Y, Stewart AF. (2001) Recombinogenics engineering-new options for cloning and manipulating DNA. Trends Biochem Sci 26: 325-331.

Poteete AR. What makes the bacteriophage lambda Red system useful for genetic engineering molecular mechanism and biological function. (2001) FEMS Microbiol Lett 201: 9-14.

Rakin A, Noelting C, Schubert S, Heesmann J. (1999) Common and specific characteristics of the high-pathogenicity island of Yersinia enterocolitica. Infect Immun 67: 5265-5274.

Schouler C, Schaeffer B, Bree A, Mora A, Dahbi G, Biet F, Oswalde E, Mainil J, Blanco J, Schouleur MM. (2012) Diagnostic strategy for identifying Avian Pathogenic Escherichia coli based on four patterns of virulence genes. J Clin Microbiol 50: 1673-1678.

Schouler C, Germon P, Chen YH, He L, Blanco JE, Bree A, Huang SH, Moulin-Schouleur M. (2005) ibeA, a virulence factor of avian pathogenic Escherichia coli. Microbiology 151: 1179-1186. 